\title{
Charmed-strange Mesons Experimental Results
}

\author{
Jianchun Wang
}

Syracuse University, Department of Physics, Syracuse, NY 13244, U.S.A.

\begin{abstract}
Two new states in the charm strange sector, $D_{s J}^{*}(2317)^{+}$and $D_{S J}(2460)^{+}$, have recently been discovered at $e^{+} e^{-}$ collider experiments. The new states are first observed in the dominant $D_{s}^{+} \pi^{0}$ and $D_{s}^{*+} \pi^{0}$ modes respectively and are very narrow. They are consistent with $0^{+}$and $1^{+} \mathrm{P}$-wave $c \bar{s}$ mesons. The $D_{s J}(2460)^{+}$meson is also observed in $D_{s}^{+} \gamma$ and $D_{s}^{+} \pi^{+} \pi^{-}$ modes. A review of the discoveries and possible explanations is given.
\end{abstract}

\section{INTRODUCTION}

In a simplified picture, the charmed-strange meson $c \bar{s}$ (generically denoted as $D_{s J}$ in this paper) is an atom of a massive charm quark and a light anti-strange quark. The mass splitting of different states is the result of interaction of the spin angular momenta of the two quarks, $\overrightarrow{s_{c}}$ and $\overrightarrow{s_{s}}$, and the orbital angular momentum $\vec{L}$ between them. According to HQET [1, 2], in the limit that the charm quark is infinitively heavy, its spin is totally decoupled from the light degree of freedom. Then the spin of charm quark $\vec{s}_{c}$ and $\vec{j}=\vec{L}+\overrightarrow{s_{s}}$ are conserved separately by strong interactions. This is the so-called heavy quark symmetry (HQS).

The charm quark, however, is not infinitively heavy, but it is heavier than the QCD scale $\Lambda_{\mathrm{QCD}}$. Thus taking $\vec{J}=\vec{L}+\vec{s}_{s}+\vec{s}_{c}$ as a good quantum number, the two ground states $\left(L=0, J^{P}=0^{-}, 1^{-}\right)$can be considered as $j=1 / 2$ doublets and the four first orbital excited states $(L=1)$ can be treated as $j=1 / 2$ doublets $\left(J^{P}=0^{+}, 1^{+}\right)$ and $j=3 / 2$ doublets $\left(J^{P}=1^{+}, 2^{+}\right)[2,3]$.

Before this year only four of these six states had been observed. All the observed ones are narrow. The $0^{-}$state, $D_{s}^{+}$, is the lightest $D_{S J}$ meson and thus can decay only weakly [4]. The $1^{-}$state, $D_{s}^{*+}$, was discovered in the electromagnetic radiative mode $D_{s}^{*+} \rightarrow D_{s}^{+} \gamma$ [5]. The kinematically allowed strong transition $D_{s}^{*+} \rightarrow D_{s}^{+} \pi^{0}$ is isospin suppressed, and has branching fraction of only $6 \%$ [6]. The two observed $L=1$ states are $D_{s 1}(2536)^{+} \rightarrow$ $D^{*} K$, and $D_{s J}(2573)^{+} \rightarrow D K[7$, 8]. Being members of $j=3 / 2$ doublets, they decay in D-wave not S-wave, explaining their relatively narrow widths.

The two missing $L=1$ states $\left(0^{+}\right.$and $\left.1^{+}\right)$were predicted by most potential models [9, 10, 11, 12, 13] to be massive enough that they would decay to $D K$ and $D^{*} K$, respectively, in a $\mathrm{S}$-wave. The widths were thus expected to be very broad, $\sim 200-300 \mathrm{MeV}$. There were,

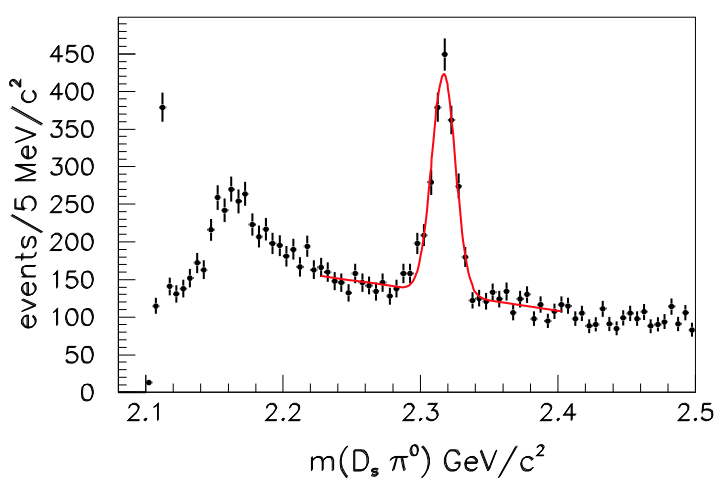

FIGURE 1. The $D_{s}^{+} \pi^{0}$ invariant mass distribution from BaBar.

however, a few predictions that these states would have masses below $D^{(*)} K$ threshold that evidently were not paid much attention [14, 15, 16]. Effectively "everyone" thought that $D^{(*)} K$ were the modes to look for these two states and they were difficult to find due to the large width. The recent discoveries reveal a different picture [17, 18, 19, 20, 21, 22].

\section{DISCOVERY OF $D_{S J}^{*}(2317)^{+}$}

The BaBar collaboration observed a $D_{c}^{+} \pi^{0}$ structure in their $e^{+} e^{-}$continuum event sample [17]. The center of peak is $2317.3 \pm 0.4 \pm 0.8 \mathrm{MeV}$ as shown in Figure 1 The width of the peak is $8.6 \pm 0.4 \mathrm{MeV}$, consistent with their detector resolution. The structure is observed in different $D_{s}^{+}$decay modes. It does not appear in their generic Monte Carlo simulated sample, and thus it is not a reflection of a previously known decay.

Since the decay products of this new state must contain a charm and an anti-strange quark, it is natural to think 


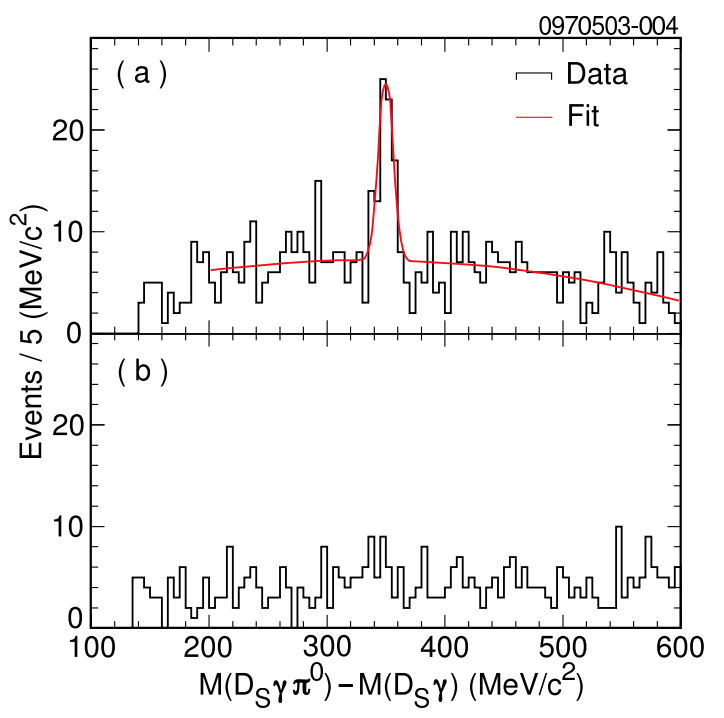

FIGURE 2. The $D_{s}^{+} \gamma \pi^{0}$ mass distribution from CLEO for $D_{s}^{+} \gamma$ candidates from a) $D_{s}^{*+}$ signal, b) $D_{s}^{*+}$ sidebands.

that this is one of the $L=1 D_{S J}$ mesons that are still missing. Thus it is named as $D_{s J}^{*}(2317)^{+}$. Furthermore, the $1^{+}$meson is forbidden to decay into $0^{-} 0^{-}$, whereas the $0^{+}$meson is allowed in S-wave. The decay angular distribution is flat after reconstruction efficiency correction, which means either $D_{s J}^{*}(2317)^{+}$is generated unpolarized or it is a spin-0 state. So this new state is probably the $0^{+}$ $D_{s J}$ meson, though higher spin is not ruled out.

The mass of $D_{s J}^{*}(2317)^{+}$, however, is much lighter than the $0^{+} D_{s J}$ meson predicted by most potential models. For example, the model in reference [13] worked quite well with known $D$ and $D_{s J}$ mesons at the time it was created, and successfully predicted the mass of $0^{+}$ and $1^{+} D$ mesons that were later discovered. It predicted the mass of $0^{+} D_{s J}$ meson to be $2487 \mathrm{MeV}$. The newly observed $D_{s J}^{*}(2317)^{+}$is $170 \mathrm{MeV}$ lower than the expectation, it is even $\sim 40 \mathrm{MeV}$ below the $D K$ threshold. And the width is much narrower $(<10 \mathrm{MeV})$ than the prediction of $\sim 200-300 \mathrm{MeV}$.

\section{DISCOVERY OF $D_{S J}(2460)^{+}$}

The CLEO collaboration confirms the $D_{s}^{+} \pi^{0}$ resonance observed by BaBar [18, 19]. They find that the measured width of the peak is $8.0_{-1.2}^{+1.3} \mathrm{MeV}$, somewhat broader than their detector resolution of $6.0 \pm 0.3 \mathrm{MeV}$. More interestingly they also observe another state, $D_{s J}(2460)^{+}$, at $2463 \mathrm{MeV}$ that decays into $D_{s}^{*+} \pi^{0}$ (Figure 2).

Figure 2 a shows the invariant mass difference, $\Delta M=$ $M\left(D_{s}^{+} \gamma \pi^{0}\right)-M\left(D_{s}^{+} \gamma\right)$. Requiring $D_{s}^{+} \gamma$ consistent with $D_{s}^{*+}$, they find $55 \pm 10$ events in the peak. The center of peak is measured to be $349.8 \pm 1.3 \mathrm{MeV}$, similar to that of $D_{s J}^{*}(2317)^{+}$that CLEO finds at $349.4 \pm 1.0 \mathrm{MeV}$ in the $\Delta M=M\left(D_{s}^{+} \pi^{0}\right)-M\left(D_{s}^{+}\right)$spectrum. The width of peak is $6.1 \pm 1.0 \mathrm{MeV}$, close to the detector resolution of $6.6 \pm 0.5 \mathrm{MeV}$. The BaBar data also shows excess in $D_{s}^{+} \gamma \pi^{0}$ invariant mass spectrum [17], although the conclusion reached in the publication was that further study is needed due to the complexity of the reflection from the $D_{S J}^{*}(2317)^{+}$.

The $\Delta M$ values are very close for $D_{s J}^{*}(2317)^{+}$and $D_{S J}(2460)^{+}$. When the $D_{s}^{+}$from a $D_{s J}^{*}(2317)^{+}$decay picks up a random photon, the invariant mass of the two can fall in the selection window of $D_{s}^{*+}$. Because of the equality of the mass difference, when the $\pi^{0}$ of the same $D_{s J}^{*}(2317)^{+}$decay is added, the total invariant mass is consistent with $D_{s J}(2460)^{+}$. Thus $D_{s J}^{*}(2317)^{+}$could reflect into $D_{S J}(2460)^{+}$peak, but simulation shows that this peak has width of $\sim 15 \mathrm{MeV}$, much broader than the real $D_{s J}(2460)^{+}$signal peak. Checking the event sample from $D_{s}^{*+}$ sidebands (Figure 2] b), CLEO find that the reflection of $D_{S J}^{*}(2317)^{+}$could only account for $1 / 5$ to $1 / 4$ of events in $D_{s J}(2460)^{+}$peak.

The reflection also exists in the opposite direction, when the single photon from $D_{S J}(2460)^{+}$decay is "ignored" and a fake $D_{s J}^{*}(2317)^{+}$peak is created. With the MC simulation event sample CLEO estimates the cross reflection efficiencies, and then extract the true number of reconstructed $D_{s J}^{*}(2317)^{+}$and $D_{S J}(2460)^{+}$signals. In both peaks, about $20 \%$ events are due to reflection. The number of $D_{s J}(2460)^{+}$signal is $41 \pm 12$, consistent with estimation using $D_{s}^{*+}$ sidebands.

The Belle collaboration confirms both $D_{s J}^{*}(2317)^{+}$ and $D_{s J}(2460)^{+}$states in continuum event sample as well as in $B$ decays that will be discussed in next section $[20,21]$. They also observed $D_{s J}(2460)^{+}$in $D_{s}^{+} \gamma$ and $D_{s}^{+} \pi^{+} \pi^{-}$modes. After careful study of cross reflection the BaBar collaboration also confirms the $D_{s J}(2460)^{+}$ meson [22]. So there is no doubt about the existence of the $D_{S J}(2460)^{+}$state. As $D_{S J}(2460)^{+}$decays to $1^{-} 0^{-}$, it is most probably the missing $J^{P}=1^{+}$state decays in a $S$ wave. It can not be a $0^{+}$state, though other possibilities are not ruled out. Further investigation is needed.

\section{OBSERVATION OF $D_{S J}^{*}(2317)^{+}$AND $D_{S J}(2460)^{+}$IN $B$ DECAYS}

Cross reflection of the two new $D_{s J}$ states in continuum data complicates the investigation. The cross reflection, however, is eliminated in $B$ decays as extra constraints are applied. Belle searches for $B \rightarrow \bar{D} D_{s J}^{+}$decays of both charged and neutral $B$ [21]. For events whose mass and beam energy constraints are consistent with the $B \rightarrow \bar{D} D_{S J}^{+}$decay, the invariant mass spectrum of 


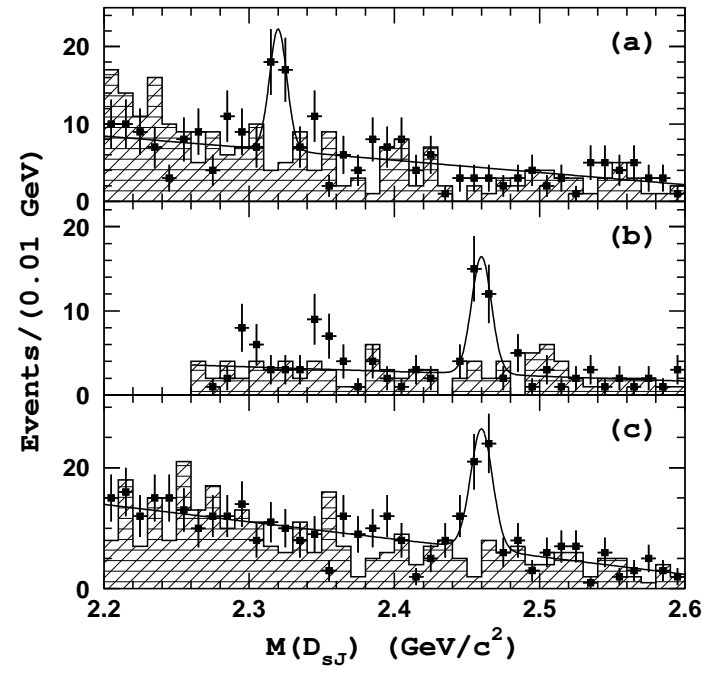

FIGURE 3. Invariant mass for $D_{S J}$ candidates produced in $B \rightarrow \bar{D} D_{s J}^{+}$decays for $D_{s J}^{+} \rightarrow$ a) $D_{s}^{+} \pi^{0}$, b) $D_{s}^{*+} \pi^{0}$ and c) $D_{s}^{+} \gamma$. The hatched regions are for $\Delta E$ sidebands.

$D_{s}^{+} \pi^{0}, D_{s}^{*+} \pi^{0}$ and $D_{s}^{+} \gamma$ are shown in Figure 3 Belle observes both the $D_{S J}^{*}(2317)^{+}$and $D_{S J}(2460)^{+}$in $B$ decays. The peak in Figure $3 \mathrm{c}$ is the first observation of $D_{s J}(2460)^{+} \rightarrow D_{s}^{+} \gamma$ mode. The ratio of partial width of this mode to that of $D_{s J}(2460)^{+} \rightarrow D_{s}^{*+} \pi^{0}$ is measured to be $0.38 \pm 0.11 \pm 0.04$, consistent with $0.55 \pm 0.13 \pm 0.8$ measured in continuum data by Belle. The branching fractions are measured to be:

$$
\begin{aligned}
\mathscr{B}\left(B \rightarrow \bar{D} D_{s J}^{*}(2317)^{+}\right) & \times \mathscr{B}\left(D_{s J}^{*}(2317)^{+} \rightarrow D_{s}^{+} \pi^{0}\right) \\
& =\left(8.5_{-1.9}^{+2.6} \pm 2.6\right) \times 10^{-4} \\
\mathscr{B}\left(B \rightarrow \bar{D} D_{s J}(2460)^{+}\right) & \times \mathscr{B}\left(D_{s J}(2460)^{+} \rightarrow D_{s}^{*+} \pi^{0}\right) \\
& =\left(17.8_{-3.9}^{+4.5} \pm 5.3\right) \times 10^{-4} \\
\mathscr{B}\left(B \rightarrow \bar{D} D_{s J}(2460)^{+}\right) & \times \mathscr{B}\left(D_{s J}(2460)^{+} \rightarrow D_{s}^{+} \gamma\right) \\
& =\left(6.7_{-1.2}^{+1.3} \pm 2.0\right) \times 10^{-4} .
\end{aligned}
$$

The $B$ decay provides a much better laboratory to study the spin parity of the new $D_{s J}$ states. In $B \rightarrow \bar{D} D_{s J}^{+}$ decay, the $D_{S J}$ is totally longitudinally polarized as both $B$ and $D$ are spin-0 particles. Belle measures the helicity angular distribution of $D_{s J}(2460)^{+}$in $D_{s}^{+} \gamma$ mode shown in Figure 4 The measurement strongly supports the $1^{+}$ assignment.

\section{POSSIBLE EXPLANATION AND SEARCH OF OTHER DECAY MODES}

The world averaged mass difference are $349.1 \pm 0.6$ $\mathrm{MeV}$ and $346.7 \pm 0.8 \mathrm{MeV}$ for $D_{s J}^{*}(2317)^{+}$and

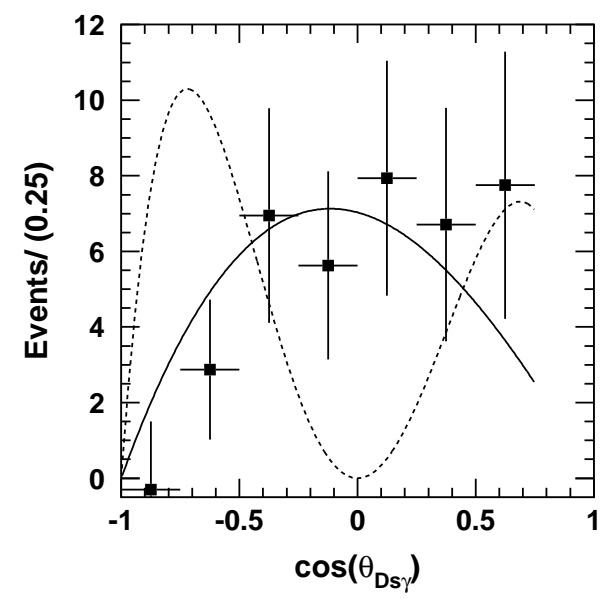

FIGURE 4. Helicity angular distribution of $D_{s J}(2460)^{+}$in $B \rightarrow \bar{D} D_{s J}(2460)^{+}, D_{s J}(2460)^{+} \rightarrow D_{s}^{+} \gamma$. The solid line is expectation of $1^{+}$state and dotted line for $2^{+}$.

$D_{S J}(2460)^{+}$respectively. Adding the PDG value of $\mathrm{M}\left(D_{s}^{+}\right)=1968.5 \pm 0.6 \mathrm{MeV}$ and $\mathrm{M}\left(D_{s}^{*+}\right)=2112.4 \pm 0.7$ $\mathrm{MeV}$, the masses are $2317.6 \pm 0.8 \mathrm{MeV}$ and $2459.1 \pm 1.0$ $\mathrm{MeV}$. The upper limits of width at $90 \% \mathrm{CL}$ are 4.6 and $5.5 \mathrm{MeV}$ respectively set by Belle [21].

Since the discovery of $D_{s J}^{*}(2317)^{+}$state several possible explanations appeared. Cahn and Jackson use nonrelativistic vector and scalar exchange forces and recalculate within potential model to explain the mass [23]. Van Beveran and Rupp use a unitarized meson model to explain the low mass as a threshold effect [24]. Bardeen et al explains that it is a normal $c \bar{s}$ state [14, 25]. Barnes et al suggest that it is a $D K$ molecule [26]. Several others propose different multi-quark models [27, 28, 29, 30, 31].

Due to the low mass and narrow width, $D_{S J}^{*}(2317)^{+}$ has difficulty fit in the potential models, nor does $D_{S J}(2460)^{+}$. They could be $D K$ and $D^{*} K$ molecules as they are about just $40 \mathrm{MeV}$ below the thresholds. The mass difference between $D$ and $D^{*}$ is $\sim 140 \mathrm{MeV}$, explaining the mass difference between $D_{S J}^{*}(2317)^{+}$and $D_{s J}(2460)^{+}$of $\sim 142 \mathrm{MeV}$. Inside the molecule, $D^{(*)}$ and $K$ are pre-formed. As the direct decay mode $D^{(*)} K$ is closed, quark antiquark pairs of the two have to be broken to form a $D_{s}^{+}$and a $\pi^{0}$, thus the decay is weak.

The molecule picture suggests the existence of $D_{s}^{(*)+} \pi^{ \pm}$resonances. Observation of these resonances would strongly support molecule hypothesis as they are not conventional $q \bar{q}$ meson due to their quark content. The CDF collaboration studies $D_{s}^{+} \pi^{ \pm}$modes and find no narrow structure. The CLEO collaboration has searched for $D_{s}^{(*)+} \pi^{ \pm}$structures as shown in Figure 5 No narrow structure is found. The productions of narrow $D_{s}^{(*)+} \pi^{ \pm}$ 


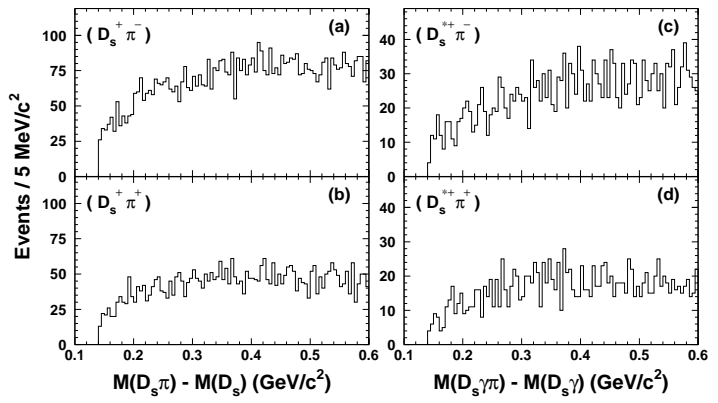

FIGURE 5. $D_{s}^{(*)+} \pi^{ \pm}$mass distribution from CLEO for a) opposite-signed $D_{s} \pi$, b) same-signed $D_{s} \pi$, c) opposite-signed $D_{s}^{*} \pi$ and d) same-signed $D_{s}^{*} \pi$.

states are at least a factor of ten lower than the $D_{s}^{(*)+} \pi^{0}$ modes. This proves that $D_{s J}^{*}(2317)^{+}$and $D_{S J}(2460)^{+}$ are iso-scalers. It, however, does not totally rule out the molecule scenario as an iso-vector molecule is expected to be broad, although there is no indication of the existence of such structure in $B$ decay sample.

The new $D_{s J}$ states fit in well the quark model as normal $0^{+}$and $1^{+} c \bar{s}$ mesons except for maybe the low masses. Bardeen et al couple chiral perturbation theory with a quark model representing HQET, and in fact predicted the masses of the $0^{+}$and $1^{+} c \bar{s}$ mesons below $D^{(*)} K$ thresholds. The narrow widths are due to isospin violation in the decays. They infer that $D_{s J}^{*}(2317)^{+}$is indeed the $0^{+} c \bar{s}$ meson. It has an $1^{+}$partner with mass splitting identical to that between $0^{-}$and $1^{-} c \bar{s}$ mesons, which is backed up by the measurements. They also calculate partial width of other decay modes as shown in Table. 11 The measured ratios and limits (at 90\% C.L.) from CLEO and Belle are also listed. The predictions are consistent with the measurements, and thus this explanation is favored.

Factorization implies that the branching fractions of $B \rightarrow \bar{D} D_{s J}^{+}$for the new $D_{S J}$ states be similar to that of $D_{s}^{+}$and $D_{s}^{*+}$, which are $\sim 1 \%$. The measurements are about a factor of ten lower. This casts a shadow on the favored conventional $c \bar{s}$ explanation. Four-quark or molecule states, however, would have branching fraction consistent with the measurements [28, 31, 32]. Browder et al propose that these states are mixtures of $c \bar{s}$ and fourquark states [33]. More experimental measurements and theoretical ideas are needed to reveal the true identity of these two new states.
TABLE 1. Ratio of branching fractions of different $D_{S J}^{*}(2317)^{+}$and $D_{S J}(2460)^{+}$modes. Limits are with 90\% CL.

\begin{tabular}{lccc}
\hline$D_{s J}^{*}(2317)^{+}$decay & BEH & Belle & CLEO \\
\hline$D_{s}^{+} \pi^{0}$ & $\equiv 1$ & $\equiv 1$ & $\equiv 1$ \\
$D_{s}^{+} \pi^{+} \pi^{-}$ & 0 & $<4 \times 10^{-3}$ & $<0.019$ \\
$D_{s}^{+} \gamma$ & 0 & $<0.05$ & $<0.052$ \\
$D_{s}^{*+} \pi^{0}$ & 0 & & $<0.01$ \\
$D_{s}^{*+} \gamma$ & 0.08 & $<0.18$ & $<0.059$ \\
\hline$D_{s J}(2460)^{+}$decay & BEH & Belle & CLEO \\
\hline$D_{s}^{*+} \pi^{0}$ & $\equiv 1$ & $\equiv 1$ & $\equiv 1$ \\
$D_{s}^{*+} \gamma$ & 0.22 & $<0.31$ & $<0.16$ \\
$D_{s}^{+} \pi^{0}$ & 0 & $<0.21$ & \\
$D_{s}^{+} \pi^{+} \pi^{-}$ & 0.20 & $0.14 \pm 0.04$ & $<0.08$ \\
$D_{s}^{+} \gamma$ & 0.24 & $0.44 \pm 0.09$ & $<0.49$ \\
$D_{s J}^{*}(2317)^{+} \gamma$ & 0.13 & & $<0.58$ \\
\hline
\end{tabular}

\section{ACKNOWLEDGMENTS}

The author would like to thank all those, especially Prof Sheldon Stone for helping prepare this note.

\section{REFERENCES}

1. De Rujula, A., Georgi, H., and Glashow, S. L., Phys. Rev. Lett., 37, 785 (1976).

2. Isgur, N., and Wise, M. B., Phys. Rev. Lett., 66, 1130 (1991).

3. Godfrey, S., and Kokoski, R., Phys. Rev., D43, 1679 (1991).

4. Chen, A., et al., Phys. Rev. Lett., 51, 634 (1983).

5. Albrecht, H., et al., Phys. Lett., B146, 111 (1984).

6. Gronberg, J., et al., Phys. Rev. Lett., 75, 3232 (1995).

7. Albrecht, H., et al., Phys. Lett., B230, 162 (1989).

8. Kubota, Y., et al., Phys. Rev. Lett., 72, 1972 (1994).

9. Godfrey, S., and Isgur, N., Phys. Rev., D32, 189 (1985).

10. Zeng, J., Van Orden, J. W., and Roberts, W., Phys. Rev., D52, 5229 (1995).

11. Gupta, S. N., and Johnson, J. M., Phys. Rev., D51, 168 (1995).

12. Ebert, D., Faustov, R. N., and Galkin, V. O. |hep-ph/9809285|.

13. Di Pierro, M., and Eichten, E., Phys. Rev., D64, 114004 (2001).

14. Bardeen, W. A., and Hill, C. T., Phys. Rev., D49, 409 (1994).

15. Fayyazuddin, and Riazuddin, Phys. Rev., D48, 2224 (1993).

16. Deandrea, A., Gatto, R., Nardulli, G., Polosa, A. D., and Tornqvist, N. A., Phys. Lett., B502, 79 (2001).

17. Aubert, B., et al., Phys. Rev. Lett., 90, 242001 (2003).

18. Stone, S., and Urheim, J., AIP Conf. Proc., 687, 96-104 (2003).

19. Besson, D., et al., Phys. Rev., D68, 032002 (2003).

20. Abe, K., et al. |hep-ex/0307052|.

21. Krokovny, P., et al. |hep-ex/0308019|. 
22. Aubert, B., et al. hep-ex/0310050|.

23. Cahn, R. N., and Jackson, J. D., Phys. Rev., D68, 037502 (2003).

24. van Beveren, E., and Rupp, G., Phys. Rev. Lett., 91, 012003 (2003).

25. Bardeen, W. A., Eichten, E. J., and Hill, C. T., Phys. Rev., D68, 054024 (2003).

26. Barnes, T., Close, F. E., and Lipkin, H. J., Phys. Rev., D68, 054006 (2003).

27. Szczepaniak, A. P., Phys. Lett., B567, 23 (2003).

28. Cheng, H.-Y., and Hou, W.-S., Phys. Lett., B566, 193 (2003).

29. Terasaki, K., Phys. Rev., D68, 011501 (2003).

30. Nussinov, S. |hep-ph/0306187|.

31. Datta, A., and O'Donnell, P. J., Phys. Lett., B567, 273 (2003).

32. Chen, C.-H., and Li, H.-n. |hep-ph/0307075|.

33. Browder, T. E., Pakvasa, S., and Petrov, A. A. |hep-ph/0307054|. 